Draft VERSION OCTOBER 10, 2018

Preprint typeset using IATEX style AASTeX6 v. 1.0

\title{
BLAZAR FLARES AS AN ORIGIN OF HIGH-ENERGY COSMIC NEUTRINOS?
}

\author{
Kohta Murase ${ }^{1,2,3,4}$, Foteini Oikonomou ${ }^{5}$, Maria Petropoulou ${ }^{6}$, \\ ${ }^{1}$ Department of Physics, Pennsylvania State University, University Park, PA 16802, USA \\ ${ }^{2}$ Department of Astronomy \& Astrophysics, Pennsylvania State University, University Park, PA 16802, USA \\ ${ }^{3}$ Center for Particle and Gravitational Astrophysics, Pennsylvania State University, University Park, PA 16802, USA \\ ${ }^{4}$ Center for Gravitational Physics, Yukawa Institute for Theoretical Physics, Kyoto University, Kyoto, Kyoto 606-8502, Japan \\ ${ }^{5}$ European Southern Observatory, Karl-Schwarzschild-Str. 2, Garching bei München D-85748, Germany \\ ${ }^{6}$ Department of Astrophysical Sciences, Princeton University, Princeton, NJ 08544, USA
}

\section{ABSTRACT}

We consider implications of high-energy neutrino emission from blazar flares, including the recent event IceCube-170922A and the 2014-2015 neutrino flare that could originate from TXS 0506+056. First, we discuss their contribution to the diffuse neutrino intensity taking into account various observational constraints. Blazars are likely to be subdominant in the diffuse neutrino intensity at sub-PeV energies, and we show that blazar flares like those of TXS $0506+056$ could make $\lesssim 1-10 \%$ of the total neutrino intensity. We also argue that the neutrino output of blazars can be dominated by the flares in the standard leptonic scenario for their $\gamma$-ray emission, and energetic flares may still be detected with a rate of $\lesssim 1 \mathrm{yr}^{-1}$. Second, we consider multi-messenger constraints on the source modeling. We show that luminous neutrino flares should be accompanied by luminous broadband cascade emission, emerging also in X-rays and $\gamma$-rays. This implies that not only $\gamma$-ray telescopes like Fermi but also X-ray sky monitors such as Swift and MAXI are critical to test the canonical picture based on the single-zone modeling. We also suggest a two-zone model that can naturally satisfy the X-ray constraints while explaining the flaring neutrinos via either photomeson or hadronuclear processes.

Keywords: astroparticle physics - galaxies: active - galaxies: jets - gamma rays: galaxies - neutrinos - radiation mechanisms: non-thermal

\section{INTRODUCTION}

Recent discoveries of high-energy cosmic neutrinos and gravitational waves have opened up a new era of multi-messenger particle astrophysics (Aartsen et al. 2013a,b; Abbott et al. 2016, 2017a,b). Whereas gravitational wave sources have been detected as individual events, no high-energy neutrino source has been confirmed so far. The observed diffuse neutrino intensity can be regarded as an isotropic neutrino background (INB) produced by a large number of sources beyond our Galaxy, because the Galactic contribution has been shown to be subdominant (for a review, see Halzen 2016). The origin of cosmic neutrinos is under active debate.

What is the fastest way to find the neutrino sources individually? Transient sources are the most promising targets, because the atmospheric background can be largely reduced by taking advantage of the time and space coincidences. The brightest transients are detectable with current detectors such as IceCube and KM3Net, even if their contribution to the INB is subdominant. Perhaps the most well-known example of neutrino-candidate transients is the prompt emission from $\gamma$-ray bursts (GRBs) with a typical duration of $\sim 1-100$ s (e.g., Waxman \& Bahcall 1997; Murase \& Nagataki 2006; Petropoulou et al. 2014; Bustamante et al. 2015), although low-power GRBs have a longer duration of $10^{3}-10^{4} \mathrm{~s}$ (Murase et al. 2006; Gupta \& Zhang 2007; Murase \& Ioka 2013). Others include GRB afterglows (Waxman \& Bahcall 2000; Murase 2007; Razzaque 2013), supernovae (Murase et al. 2011; Murase 2018; Petropoulou et al. 2017), tidal disruption events (e.g., Murase 2008; Wang et al. 2011), microquasars (e.g., Levinson \& Waxman 2001; Distefano et al. 2002; Torres et al. 2005), and blazar flares (e.g., Bednarek \& Protheroe 1999; Atoyan \& Dermer 2001; Halzen \& Hooper 2005; Dermer et al. 2012, 2014; Petropoulou et al. 2016; Gao et al. 2017).

Blazars, a subclass of active galactic nuclei (AGNs) with relativistic jets pointing toward the observer (Urry \& Padovani 1995), and their misaligned counterpart, radio galaxies, have been discussed as the sources of ultrahigh-energy cosmic rays (UHECRs) and/or high-energy neutrinos (see Murase 2017, for 
a review). Blazars are classified into BL Lac objects (BL Lacs) and quasar-hosted blazars that are mostly flat-spectrum radio quasars (FSRQs). Blazars can also be divided into high-synchrotron-peaked (HSP), intermediate-synchrotron-peaked (ISP), and low-synchrotron-peaked (LSP) objects. The acceleration and survival of UHECR nuclei are possible in BL Lacs (Murase et al. 2012b; Rodrigues et al. 2018), whereas efficient photodisintegration and neutrino production are expected in FSRQs (Murase et al. 2014; Palladino et al. 2018).

Recently, IceCube-Collaboration et al. (2018) have reported a $\sim 0.1-1 \mathrm{PeV}$ muon neutrino event, IceCube$170922 \mathrm{~A}$, coincident with a month- to year-long $\gamma$-ray flare of the blazar TXS $0506+056$ at redshift $z \approx$ 0.336 (Paiano et al. 2018). The public alert was sent via the Astrophysical Multi-messenger Network Observatory $(\mathrm{AMON})$, and the follow-up searches led to the discovery of GeV-TeV $\gamma$-ray counterparts as well as Xray and optical emission (IceCube-Collaboration et al. 2018; Keivani et al. 2018). Furthermore, the archival search of the past IceCube data revealed $13 \pm 5$ signals of lower-energy muon neutrinos coming from the same region in the sky on a time scale of 5 months (IceCube-Collaboration 2018). Although it is still too early to be conclusive about their physical association, the reported significance of $\sim 3 \sigma-4 \sigma$ is interesting enough to make us discuss the implications of the neutrino flare-blazar connection.

IceCube-170922A and the 2014-2015 neutrino flare: The neutrino energy estimated for IceCube-170922A is $E_{\nu} \sim 0.3 \mathrm{PeV}$, and the $p$-value (chance probability) for the coincidence with the flare from the ISP/LSP blazar, TXS $0506+56$, is $\sim 0.3 \%$, corresponding to a significance of $\approx 3 \sigma$ (IceCube-Collaboration et al. 2018). The neutrino flare found in the lower-energy data prior to the discovery of IceCube-170922A has a significance of $\approx 3.5 \sigma$ (IceCube-Collaboration 2018). The inferred muon neutrino energy fluences are $E_{\nu}^{2} \phi_{\nu_{\mu}} \sim 10^{-4}-$ $10^{-3} \mathrm{erg} \mathrm{cm}^{-2}$, implying a released neutrino energy of $\mathcal{E}_{\nu}^{\mathrm{fl}} \sim 10^{53}-10^{54} \mathrm{erg}$. With a flare duration of $t_{\text {dur }} \sim 10^{7} \mathrm{~s}$, the flaring neutrino luminosity is estimated to be $L_{\nu}^{\mathrm{f}} \sim 10^{46}-10^{47} \mathrm{erg} \mathrm{s}^{-1}$, comparable to the $\gamma$-ray luminosity of the 2017 flare of TXS $0506+56$, $L_{\gamma}^{\mathrm{fl}} \sim 2 \times 10^{47} \mathrm{erg} \mathrm{s}^{-1}$ at $0.1-300 \mathrm{GeV}$.

IceCube-160731: A high-energy track event with an energy higher than several hundred $\mathrm{TeV}$ was coincident with the $\gamma$-ray counterpart detected by AGILE, AGL J1418+0008 (Lucarelli et al. 2017). The $\gamma$-rays were seen $1-2$ days before IceCube-160731, with a possible association with the BL Lac object, 1RXS J141658.0001449.

Big Bird (HESE-35): This high-energy starting event had a deposited energy of $2 \mathrm{PeV}$, which could be associated with the FSRQ, PKS B1424-418 at $z=1.522$.
Whereas the angular uncertainty for such shower events is $\sim 10^{\circ}-15^{\circ}$, the $p$-value for the coincidence was 0.05 (Kadler et al. 2016). If this association is physical, the estimated neutrino luminosity is $L_{\nu} \gtrsim 3 \times$ $10^{48} \mathrm{erg} \mathrm{s}^{-1}$.

This work focuses on implications of IceCube-170922A and the 2014-2015 neutrino flare, assuming that the association with TXS $0506+056$ is physical. We first examine the connection between blazars and the INB (Sec. 2), and argue that neutrino flares like the ones observed from TXS 0506+056 in 2017 and 2014-2015 are likely to be rare and bright events. We then show that $\mathrm{X}$-ray observations are critical in testing the standard blazar scenario for neutrino emission and for explaining either flare event observed from this blazar, and we discuss possible multizone models in Section 3. We conclude in Section 4.

\section{CONTRIBUTION TO THE INB}

Here we discuss existing constraints on the blazar contribution to the INB, which are obtained for timeaveraged emission. Given that blazars are variable sources across the electromagnetic spectrum, we then investigate the contribution of blazar flares to the INB.

\subsection{General Constraints}

The blazar contribution to the INB has been constrained by different types of analyses: (i) diffuse searches for extremely high-energy (EHE) neutrinos (Aartsen et al. 2016, 2017a), (ii) event clustering and autocorrelation analyses (Murase \& Waxman 2016; Aartsen et al. 2015, 2017b), and (iii) stacking and crosscorrelation analyses (Aartsen et al. 2017c,b).

Neutrino spectra have been predicted by most theoretical models to be hard and peaking at energies beyond $1 \mathrm{PeV}$. The hardness of the spectrum is related to the fact that the target photon density in blazars is higher at lower energies. Upon normalization to the IceCube flux at $\sim 1 \mathrm{PeV}$, most model-predicted fluxes at $10 \mathrm{PeV}$ are found to be $E_{\nu}^{2} \Phi_{\nu} \gtrsim(3-5) \times$ $10^{-8} \mathrm{GeV} \mathrm{cm}^{-2} \mathrm{~s}^{-1} \mathrm{sr}^{-1}$ for all flavors (Murase 2017, and references therein). With the $9 \mathrm{yr}$ diffuse analysis, the IceCube Collaboration reported an upper limit on the INB, $E_{\nu}^{2} \Phi_{\nu} \lesssim 1 \times 10^{-8} \mathrm{GeV} \mathrm{cm}^{-2} \mathrm{~s}^{-1} \mathrm{sr}^{-1}(90 \%$ CL) at 5-10 PeV (Aartsen et al. 2017a), excluding some of the optimistic physical models for blazar neutrino emission (see also Neronov et al. 2017).

Another type of less model-dependent constraints is obtained from the absence of sources of high-energy multiplets (Murase \& Waxman 2016; Ahlers \& Halzen 2014) (see also Lipari 2008; Silvestri \& Barwick 2010; Murase et al. 2012a, for earlier works). Let the number of the sources with multiplets be denoted as $N_{m \geq k}$. Then, constraints can be placed by requiring $N_{m \geq k} \leq 1$ at sufficiently high energies (e.g., $>50 \mathrm{TeV}$ for muons). 
Although the energy-dependent effective area should be taken into account for detailed calculations as in Murase \& Waxman (2016), the basic results can be understood by using a limit from the nondetection of point sources. The 8 yr point-source sensitivity (90\% CL) for an $E^{-2}$ neutrino spectrum is $F_{\lim } \sim(5-6) \times$ $10^{-10} \mathrm{GeV} \mathrm{cm}^{-2} \mathrm{~s}^{-1}$ (Aartsen et al. 2017b). For such a flat energy spectrum with a time-averaged luminosity of $\varepsilon_{\nu} L_{\varepsilon_{\nu \mu}}^{\text {ave }} \sim 10^{44} \mathrm{erg} \mathrm{s}^{-1}$, the number density of the sources is constrained as:

$$
\begin{aligned}
n_{0}^{\mathrm{eff}} & \lesssim 1.9 \times 10^{-10} \mathrm{Mpc}^{-3}\left(\frac{\varepsilon_{\nu} L_{\varepsilon_{\nu_{\mu}}}^{\mathrm{ave}}}{10^{44} \mathrm{erg} \mathrm{s}^{-1}}\right)^{-3 / 2} \\
& \times\left(b_{m} q_{L} / 6.6\right)^{-1} F_{\mathrm{lim},-9.2}^{3 / 2}\left(\frac{2 \pi}{\Delta \Omega}\right),
\end{aligned}
$$

where $q_{L} \sim 1-3$ is a luminosity-dependent correction factor determined by the redshift evolution and $\Delta \Omega$ is the solid angle covered by the detector. This limit depends on spectral templates of the sources, and the differential sensitivity can be worse than the integrated sensitivity by $\sim \ln (10)$. Thus, for harder spectra explaining neutrinos only in the $\mathrm{PeV}$ range (see Figs. 2 and 4 of Murase \& Waxman 2016), the upper limit in Eq. (1) can be relaxed by $\sim 3$. Note that $b_{m} \geq 1$ is a factor that represents details of the analysis. For example, in the case of the multiplet analysis, we have $b_{m} \approx 6.6$ for $m \geq 2$ and $b_{m} \approx 1.6$ for $m \geq 3$.

Using Eq. (1) we derive an upper limit on the contribution of a source population with $n_{0}^{\text {eff }}$ and to the INB:

$$
\begin{aligned}
E_{\nu}^{2} \Phi_{\nu} & \approx \frac{\xi_{z} c t_{H}}{4 \pi} 3\left(\varepsilon_{\nu} L_{\varepsilon_{\nu \mu}}^{\mathrm{ave}}\right) n_{0}^{\mathrm{eff}} \\
& \lesssim 6.9 \times 10^{-9} \mathrm{GeV} \mathrm{cm}^{-2} \mathrm{~s}^{-1} \mathrm{sr}^{-1}\left(\frac{\xi_{z}}{0.7}\right)\left(\frac{6.6}{b_{m} q_{L}}\right)^{2 / 3} \\
& \times\left(\frac{n_{0}^{\mathrm{eff}}}{10^{-7} \mathrm{Mpc}^{-3}}\right)^{1 / 3} F_{\mathrm{lim},-9.2}\left(\frac{2 \pi}{\Delta \Omega}\right)^{2 / 3}
\end{aligned}
$$

where $t_{H}$ is the Hubble time and $\xi_{z}$ represents the redshift evolution of the neutrino luminosity density of the sources: $\xi_{z} \sim 0.7$ for the $\gamma$-ray luminosity density evolution of BL Lacs, $\xi_{z} \sim 8$ for that of FSRQs, and $\xi_{z} \sim 3$ for the X-ray luminosity density evolution of AGNs (Ajello et al. 2014; Ueda et al. 2014). Note that if one uses the number density evolution (corresponding to the equal luminosity weight), we have $\xi_{z} \sim 0.2$ for all BL Lacs with $n_{s} \propto(1+z)^{-3.5}$ and $\xi_{z} \sim 0.1$ for HSP objects with $n_{s} \propto(1+z)^{-6}$, respectively (Ajello et al. 2014). In the extreme case where the faintest BL Lacs are equally neutrino emitters, we have $n_{0}^{\text {eff }}=n_{0}^{\text {tot }} \sim(1-3) \times 10^{-7} \mathrm{Mpc}^{-3}$ (Ajello et al. 2014) and $\xi_{z} \sim 0.2$, implying that the contribution to the INB is $\lesssim 10 \%$ at $0.1 \mathrm{PeV}$. Although the limit could be further relaxed by a factor of two by integrating the number density down to the faintest source tail, blazars are unlikely to be dominant in the INB for such weak redshift evolution. One should keep in mind that the effective number density, which depends on the neutrino luminosity function (i.e., $d n_{s} / d L_{\nu}$ ), should always be smaller than the total number density, i.e., $n_{0}^{\text {eff }}<n_{0}^{\text {tot }}$ (Murase \& Waxman 2016). This is because physical models typically predict $L_{\nu} \propto L_{\gamma}-$ $L_{\gamma}^{2}$ (Murase et al. 2014; Tavecchio \& Ghisellini 2015; Petropoulou et al. 2015; Murase \& Waxman 2016). In the fiducial case of the leptonic scenario for BL Lacs, the effective number density is $n_{0}^{\text {eff }} \sim 10^{-9}-10^{-8} \mathrm{Mpc}^{-3}$, which gives $\lesssim(5-10)\left(\xi_{z} / 0.7\right) \%$ of the INB. For FSRQs, we have $n_{0}^{\text {eff }} \sim 10^{-12}-10^{-11} \mathrm{Mpc}^{-3}$, leading to $\lesssim(6-10)\left(\xi_{z} / 8\right) \%$ in the $0.1 \mathrm{PeV}$ range. Note that the neutrino luminosity density evolution would be stronger than the $\gamma$-ray one if the $\gamma$-ray luminosity more strongly weighs on the neutrino luminosity, but such cases are constrained by the stacking limits. In this sense these constraints are complementary to each other.

Another limit can be placed by the autocorrelation analysis on the small-scale anisotropy (Aartsen et al. 2015, 2017b; Ando et al. 2017). With the measured INB and the latest anisotropy limit (Aartsen et al. 2017b), the upper limit on the Poisson angular spectrum is estimated to be $E_{\nu}^{4} C_{P}<4 \times 10^{-19} \mathrm{GeV}^{2} \mathrm{~cm}^{-2} \mathrm{~s}^{-2} \mathrm{sr}^{-1}$. Then, using the known formula of $C_{P}$ for standard candle sources, we obtain $n_{0}^{\text {eff }} \lesssim 1.1 \times$ $10^{-9} \mathrm{Mpc}^{-3}\left(\varepsilon_{\nu} L_{\varepsilon_{\nu \mu}}^{\mathrm{eff}} / 10^{44} \mathrm{erg} \mathrm{s}^{-1}\right)^{-3 / 2} q_{L}^{-1}(2 \pi / \Delta \Omega)^{1 / 4}$, which gives a comparable limit.

Blazars are highly variable objects, and as a result, their luminosity density could be dominated by the flaring states characterized by a "flaring" neutrino luminosity $L_{\nu}^{\mathrm{fl}}$. In this "flare-dominated" case, the average neutrino luminosity can be written as $L_{\nu}^{\text {ave }} \approx f_{\mathrm{fl}} L_{\nu}^{\mathrm{f}}$, where $f_{\mathrm{ff}}$ is the duty factor of flares, which will be discussed in the next subsection. The differential neutrino luminosity density is then written as $\varepsilon_{\nu} Q_{\varepsilon_{\nu}}=$ $\left(\varepsilon_{\nu} L_{\varepsilon_{\nu}}^{\mathrm{ave}}\right) n_{0}^{\mathrm{eff}} \approx\left(\varepsilon_{\nu} L_{\varepsilon_{\nu}}^{\mathrm{fl}}\right)\left(f_{\mathrm{fl}} n_{0}^{\mathrm{eff}}\right)$. For transients, including flaring sources, the atmospheric background can be reduced owing to the shorter time window, thereby improving, in general, the fluence sensitivity. The power of such a time-dependent search was demonstrated in IceCube-Collaboration (2018) (although the excess besides IceCube-170922A was not significant in the timeintegrated search). Thus, Eq. (1) for the time-averaged emission can still be regarded as a "conservative" limit on flaring sources, and the constraint given by Eq. (2) is still applicable even if blazars are highly variable ${ }^{1}$.

\footnotetext{
1 Note that multiplet limits are applicable to transients, and we stress that such a "transient" case is here considered. Indeed, even if the 2014 flare of TXS 0506+056 is included, $N_{m \geq k}$ for $\gtrsim 50 \mathrm{TeV}$ muons seems no more than one, leading to, e.g., $\rho_{0}^{\text {eff }} \gtrsim 1.7 \times$ $10^{4} \mathrm{Gpc}^{-3} \mathrm{yr}^{-1}\left(b_{m} q_{L} / 6.6\right)^{2}(\Delta \Omega / 2 \pi)^{2}\left(T_{\mathrm{obs}} / 8 \mathrm{yr}\right)^{2}\left(\xi_{z} / 0.7\right)^{-3} \phi_{\mathrm{lim},-1}^{-3}$ $\max \left[N_{\mathrm{fl}}, 1\right]$ for transients accounting for all the diffuse flux. Here $N_{\mathrm{fl}} \approx f_{\mathrm{fl}} T_{\mathrm{obs}} / t_{\mathrm{dur}} \approx T_{\mathrm{obs}} / \Delta T_{\mathrm{fl}}$ is the number of flaring peri-
} 
As noted above, stacking and cross-correlation analyses can provide tighter constraints especially for physically motivated models. In particular, for the $2 \mathrm{LAC}$ catalog consisting of 862 blazars, the blazar contribution to the INB is restricted to be $\leq(19-27) \%$ (Aartsen et al. 2017c). Note that theory typically predicts $L_{\nu} \propto$ $L_{\gamma}-L_{\gamma}^{2}$ (Murase et al. 2014; Tavecchio \& Ghisellini 2015; Petropoulou et al. 2015), and a flux weighting with $F_{\nu} \propto F_{\gamma}$ leads to $\lesssim 7 \%$ (Aartsen et al. 2017c). For HSP BL Lacs, the preliminary result gives $\leq$ $(4.5-5.7) \%$ (Aartsen et al. 2017b) in the equal flux weight $\left(F_{\nu} \propto\right.$ const. $)$ assumption. The stacking limits are powerful and more meaningful when the physical luminosity weight is strong (in which the neutrinos mostly come from the resolved blazars; see Murase et al. 2014). Thus, $\gamma$-ray-bright blazars that significantly contribute to the extragalactic $\gamma$-ray background are disfavored as the dominant $(\sim 100 \%)$ origin of IceCube's neutrinos (see also Wang \& Li 2016; Zhang \& Li 2017; Neronov et al. 2017; Palladino \& Vissani 2017; Ando et al. 2017).

All the constraints discussed so far can be relaxed by a factor of a few under different assumptions. (i) The diffuse EHE limits can be avoided if the cosmicray $(\mathrm{CR})$ spectrum is sufficiently soft or the maximum CR energy is lower than $\sim 10-100 \mathrm{PeV}$ (far below UHECR energies), as considered in Murase et al. (2014) and Dermer et al. (2014). This is because the neutrino production efficiency increases with energy, and the resulting neutrino spectra are hard for a power-law CR spectrum with $s_{\text {cr }} \sim 2-2.6$. (ii) The multiplet limit is sensitive to $\xi_{z}$. Weakly evolving sources such as BL Lacs are strongly constrained. On the other hand, rapidly evolving sources such as FSRQs could give a significant contribution to the INB if $n_{0}^{\text {eff }} \sim n_{0}^{\text {tot }} \sim 10^{-9} \mathrm{Mpc}^{-3}$. However, this is contrary to the fiducial theoretical prediction, $n_{0}^{\text {eff }} \ll n_{0}^{\text {tot }}$. Dermer et al. (2014) proposed such a model, in which flaring blazars significantly contribute to the INB only in the $\mathrm{PeV}$ range, but this model does not explain the UHECRs (see also Murase 2017). (iii) The stacking limits do not apply to $\gamma$-ray dark blazars. For example, a subset of FSRQs with a spectral energy distribution ( $\mathrm{SED}$ ) peak in the $\mathrm{MeV}$ range are dim in the Fermi LAT band, and such MeV blazars could sig-

ods and $\Delta T_{\mathrm{f}}$ is the typical flare interval. When at least one flare occurs in the observation time (i.e., $\Delta T_{\mathrm{fl}} \lesssim T_{\text {obs }}$ ), the expressions for density and diffuse limits on flaring neutrino sources eventually become similar to those for steady sources, and one can easily obtain Eqs. (1) and (2). Here substituting the time-averaged sensitivity gives conservative results because of $F_{\text {lim }}>\phi_{\text {lim }} / T_{\text {obs }}$, but this does not mean that we assume steady sources. The constraints for rare transients such that $\Delta T_{\mathrm{fl}} \gtrsim T_{\mathrm{obs}}$, including non-repeating ones, can be obtained by replacing $n_{0}^{\text {eff }}$ in Eq. (2) with $\rho_{0}^{\text {eff }} T_{\text {obs }} \approx n_{0}^{\text {eff }}\left(T_{\text {obs }} / \Delta T_{\mathrm{fl}}\right)$. Because of $n_{0}^{\text {eff }}\left(T_{\text {obs }} / \Delta T_{\mathrm{fl}}\right) \lesssim n_{0}^{\text {eff }}$, Eq. (2) still gives a conservative limit on the diffuse flux contribution. nificantly contribute to the INB as hidden CR accelerators (Murase et al. 2016).

The combination of all constraints indicates that the blazar contribution to the INB is likely to be subdominant at least in the $0.1 \mathrm{PeV}$ range. This is even more so the case for the medium-energy component in the $10-100 \mathrm{TeV}$ range, which requires models prohibiting the escape of $\gamma$-rays (Murase et al. 2016). On the other hand, at present, the contribution could be more significant in the $\mathrm{PeV}$ or higher-energy range. We note that fiducial models (normalized to the UHECR flux), presented in Murase et al. (2014), are consistent with the above constraints, and give $\sim 2-10 \%$ of the INB in the $0.1 \mathrm{PeV}$ range (and more at higher energies) .

\subsection{Implications of TXS 0506+056}

Bright flaring sources are detectable in neutrinos whether the blazars are dominant or subdominant in the extragalactic neutrino sky (Dermer et al. 2014; Murase \& Waxman 2016; Guépin \& Kotera 2017). Nevertheless, it is natural to estimate the contribution of blazar flares like the ones from TXS $0506+056$ to the INB and discuss the detectability of similar flaring events.

The flaring state lasts only for a fraction of the observation time. For a given time binning, one can measure the number of detected particles (e.g., photons) per bin, which is proportional to luminosity. We introduce the flaring state when the number of photons in a bin exceeds a certain threshold corresponding to the luminosity $L^{\text {th }}$. Then one can construct the distribution of the number of time bins with luminosity, $d N / d L$. The fraction of time spent in the flaring state (i.e., the duty factor) is given by

$$
f_{\mathrm{fl}}=\frac{1}{N_{\mathrm{tot}}} \int_{L^{\mathrm{th}}} d L \frac{d N}{d L},
$$

where $N_{\text {tot }}$ is the total number of time bins. The fraction of energy emitted in the flaring state is:

$$
b_{\mathrm{fl}}=\frac{1}{L^{\text {ave }} N_{\mathrm{tot}}} \int_{L^{\mathrm{th}}} d L L \frac{d N}{d L},
$$

where the average luminosity is given by $L^{\text {ave }}=$ $\left(1 / N_{\text {tot }}\right) \int d L L(d N / d L)$ and the average flaring luminosity is rewritten as $L^{\mathrm{fl}}=\left(b_{\mathrm{fl}} / f_{\mathrm{fl}}\right) L^{\text {ave }}$. Note that in the flare-dominated limit, $b_{\mathrm{fl}} \approx 1$, we have $L^{\text {ave }} \approx f_{\mathrm{fl}} L^{\mathrm{fl}}$.

Using the public Fermi All-sky Variability Analysis (FAVA) data by Fermi LAT (Abdollahi et al. 2017), one can obtain the luminosity distribution, $d N / d L_{\gamma}$ of a source, under the assumption that the spectral shape does not change during flaring states (since only photon counts are available in the FAVA analysis). In the latter case, $d N / d L_{\gamma} \propto d N / d N_{\gamma}$. Henceforth, we use $d N / d N_{\gamma}$ and $d N / d L_{\gamma}$ interchangeably. An example for TXS $0506+056$ is shown in Fig. 1, in which the photon distribution is modeled as a power law with slope 


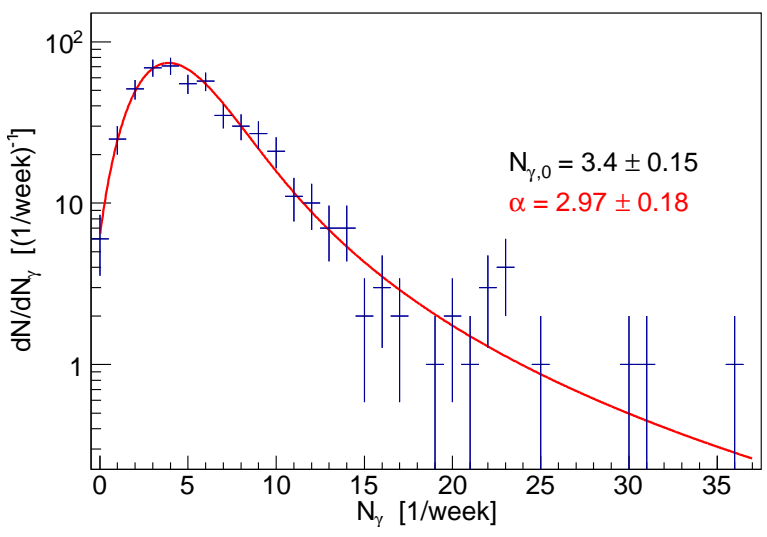

Figure 1. Histogram of the number of photons, $N_{\gamma}$ detected per week by the FAVA analysis in the direction of TXS $0506+056$ in the high-energy bin $(800 \mathrm{MeV}-300 \mathrm{GeV})$. The photon distribution is modeled as a power law with spectral index $\alpha$ above a minimum "quiescent" count rate $N_{\gamma, 0}$ (red solid line). The error bars are statistical.

$\alpha$ above a minimum number of photons per bin $N_{\gamma, 0}$ that corresponds to a "quiescent" flux. The number of detected photons per time interval is then given by a convolution of this power law with a Poissonian distribution. We applied the same method to a selection of BL Lacs at intermediate redshifts from FAVA, and find that they are well described by a power-law $d N / d L_{\gamma} \propto L_{\gamma}^{-\alpha}$ with $\alpha \sim 2-4$. One can also calculate $f_{\mathrm{fl}}$ and $b_{\mathrm{fl}}$ for various blazars using Eqs. (3) and (4) (see Table 1). The exact values depend on the definition of the flaring state (see, e.g., Resconi et al. 2009), but our main conclusions are not expected to change, if flares are defined differently. We found that the duty factor lies in the range of $0.3-10 \%$ for $\geq 5 \sigma$ flares (as per the FAVA definition), and obtained $f_{\mathrm{fl}} \approx 0.02-0.1$ for TXS $0506+056$. The corresponding fraction of emitted photons is $b_{\mathrm{fl}} \sim 0.1 \ll 1$, implying that the $\gamma$-ray emission is dominated by steady emission. Although a detailed statistical study is beyond the purpose of the present work, we underline the need for a systematic study of the properties of flaring, $\gamma$-ray-bright blazars. For the purposes of the present work, we treat TXS $0506+056$ as a "characteristic" test case.

During the flare of TXS $0506+056$ in the period 2017 September 15-27, the $1-100 \mathrm{GeV}$ "differential" $\gamma$ ray luminosity $\left(\varepsilon_{\gamma} L_{\varepsilon_{\gamma}}^{\mathrm{fl}} \sim 2 \times 10^{46} \mathrm{erg} \mathrm{s}^{-1}\right)$ was about 6 times higher than the average in the 3FGL cata$\log \left(\varepsilon_{\gamma} L_{\varepsilon_{\gamma}}^{\text {ave }} \sim 4 \times 10^{45} \mathrm{erg} \mathrm{s}^{-1}\right.$ ) (Tanaka et al. 2017; Acero et al. 2015). ${ }^{2}$ Thus this flare can be regarded as

\footnotetext{
2 In the Fermi ATel, a different energy range is used for the Fermi analysis $(0.1-300 \mathrm{GeV})$, giving $L_{\gamma, 0.1-300 \mathrm{GeV}}^{\mathrm{fl}} \sim 2 \times$ $10^{47} \mathrm{erg} \mathrm{s}^{-1}$ for the $\gamma$-ray luminosity of TXS $0506+056$ during the flare. Correspondingly for the quiescent luminosity in the $3 \mathrm{FGL}$ catalogue we obtain $L_{\gamma, 0.1-300 \mathrm{GeV}}^{\text {ave }} \sim 3 \times 10^{46} \mathrm{erg} \mathrm{s}^{-1}$ from the flux estimate of Tanaka et al. (2017) assuming an unbroken power-
}

Table 1. Flare Duty Factors $\left(f_{\mathrm{f}}\right)$ and Flare Energy Fractions $\left(b_{\mathrm{fl}}\right)$ for TXS 0506+056, OJ 287, PKS 0426-380, PKS 0301-243, S5 0716+071 and S4 0954+065 as Derived from the FAVA Analysis (Abdollahi et al. 2017).

\begin{tabular}{|c||c|c|c|c|c|c|}
\hline Name & $L_{\gamma}$ & $f_{\mathrm{fE}}^{\mathrm{LE}}$ & $f_{\mathrm{fl}}^{\mathrm{HE}}$ & $b_{\mathrm{f}}^{\mathrm{LE}}$ & $b_{\mathrm{fl}}^{\mathrm{HE}}$ & $\alpha$ \\
\hline TXS 0506+056 & $10^{46.3}$ & 0.1 & 0.03 & 0.1 & 0.1 & 3.0 \\
\hline OJ 287 & $10^{46.1}$ & 0.02 & 0.01 & 0.04 & 0.1 & 2.9 \\
\hline PKS 0426-380 & $10^{48}$ & 0.1 & 0.1 & 0.2 & 0.2 & 1.7 \\
\hline PKS 0301-243 & $10^{46}$ & 0.04 & 0.05 & 0.1 & 0.3 & 2.5 \\
\hline S5 0716+071 & $10^{46.7}$ & 0.07 & 0.08 & 0.1 & 0.2 & 1.7 \\
\hline S4 0954+065 & $10^{45.5}$ & 0.04 & 0.03 & 0.07 & 0.3 & 2.5 \\
\hline
\end{tabular}

Note. Values are reported for the low-energy (LE: $100-$ $800 \mathrm{MeV}$ ) and high-energy (HE: $800 \mathrm{MeV}-300 \mathrm{GeV}$ ) bins. The duty factors quoted are for flaring periods when the flux is enhanced by $\geq 5 \sigma$ according to the FAVA definition. The "integrated" $\gamma$-ray luminosity of the sources, $L_{\gamma}$ (in units of $\mathrm{erg} \mathrm{s}^{-1}$ ), is derived from the 3FGL in the $1-100 \mathrm{GeV}$ energy range. Rounded values of the power-law index $(\alpha)$ are also shown.

one of the brightest flares of this object. Note that the $\gamma$-ray photon index in the $0.1-300 \mathrm{GeV}$ range had a similar value during the September flare, $\beta=2.0$, to that in the 3FGL.

Naively, high-energy neutrino emission is dominated by the non-flaring contribution, if one assumes $L_{\nu} \propto$ $L_{\gamma}$. However, in the standard leptonic scenario for the blazar $\gamma$-ray emission, flares can dominate the neutrino output of a blazar. Under different assumptions, the leptonic models predict $L_{\nu} \propto L_{\gamma}^{\gamma}$ with $\gamma \sim 1.5-2$ (see Murase \& Waxman 2016, and references therein), giving

$$
L_{\nu}^{2} \frac{d N}{d L_{\nu}} \propto L_{\nu}^{1-\frac{\alpha-1}{\gamma}}
$$

which implies that the flaring contribution can be dominant, e.g., for BL Lacs with $\gamma \sim 2$ and $\alpha \leq 3$. Such a situation might be the case for the multi-messenger flare associated with IceCube-170922A. In addition, the neutrino flare emission can be pronounced if the CR spectrum is harder during the high state. To demonstrate this, let us consider a toy model where the low-state and high-state CR spectra are described as $\varepsilon_{p} L_{\varepsilon_{p}}^{l} \propto \varepsilon_{p}^{2-s_{l}}$ (for $s_{l}>2$ ) and $\varepsilon_{p} L_{\varepsilon_{p}}^{\mathrm{fl}} \propto \varepsilon_{p}^{2-s_{\mathrm{fl}}}$ (for $s_{\mathrm{fl}}<2$ ), respectively. Assuming that the maximum and minimum energies are $\varepsilon_{p}^{\max }$ and $\varepsilon_{p}^{\min }$, the flux enhancement at $\varepsilon_{\nu}$ is:

$c\left[\varepsilon_{\nu}\right] \equiv \frac{\varepsilon_{\nu} L_{\varepsilon_{\nu}}^{\mathrm{fl}}}{\varepsilon_{\nu} L_{\varepsilon_{\nu}}^{l}} \approx \frac{\left(2-s_{\mathrm{fl}}\right) f_{p \gamma}^{\mathrm{fl}}}{\left(s_{l}-2\right) f_{p \gamma}^{l}}\left(\frac{20 \varepsilon_{\nu}}{\varepsilon_{p, \max }^{\mathrm{f}}}\right)^{2-s_{\mathrm{fl}}}\left(\frac{20 \varepsilon_{\nu}}{\varepsilon_{p, \min }^{l}}\right)^{s_{l}-2}$,

where $f_{p \gamma}$ is the effective photomeson production optical depth that we will discuss below. If we adopt indicative

law with index $\beta=2.0$. 
values, namely, $s_{l} \sim 2.3, s_{\mathrm{fl}} \sim 1.8, \varepsilon_{\nu} \sim 0.05 \varepsilon_{p, \max }^{\mathrm{fl}} \sim$ $0.1 \mathrm{PeV}$, and $\varepsilon_{p, \text { min }}^{l} \sim 10 \mathrm{GeV}$ we find $c\left[\varepsilon_{\nu}\right] \sim 30 f_{p \gamma}^{\mathrm{fl}} / f_{p \gamma}^{l}$. Unless the physical conditions during flares change so radically that $f_{p \gamma}^{\mathrm{fl}} / f_{p \gamma}^{l} \ll 1$, flares may dominate the neutrino output of a blazar. Such a situation could be realized in the 2014-2015 neutrino flare. For either case, in the flare-dominated regime, the time-averaged neutrino luminosity can be written as $\varepsilon_{\nu} L_{\varepsilon_{\nu}}^{\text {ave }} \approx f_{\mathrm{fl}}\left(\varepsilon_{\nu} L_{\varepsilon_{\nu}}^{\mathrm{f}}\right)$.

From the above considerations, neutrinos can be copiously produced during the high states. In particular, the contribution of blazar flares like those observed from TXS $0506+056$ to the INB is constrained as:

$$
\begin{aligned}
E_{\nu}^{2} \Phi_{\nu} & \lesssim 3.8 \times 10^{-10} \mathrm{GeV} \mathrm{cm}^{-2} \mathrm{~s}^{-1} \mathrm{sr}^{-1}\left(\frac{2 \pi}{\Delta \Omega}\right)\left(\frac{\xi_{z}}{0.7}\right) \\
& \times\left(\frac{0.05}{f_{\mathrm{fl}}}\right)^{1 / 2}\left(\frac{10^{46} \mathrm{erg} \mathrm{s}^{-1}}{\varepsilon_{\nu} L_{\varepsilon_{\nu \mu}}^{\mathrm{fl}}}\right)^{1 / 2}\left(\frac{6.6}{b_{m} q_{L}}\right) F_{\mathrm{lim},-9.2}^{3 / 2},(7)
\end{aligned}
$$

where Eq. (2) is used. They can contribute up to a few percent of the INB, allowing uncertainties in the redshift evolution and neutrino spectrum. Eq. (7) also implies that dimmer blazar flares could make a larger contribution, but the total neutrino intensity must not exceed the upper limit given by Eq. (2).

Finally, we estimate how many bright flares can be detected with multi-messenger (neutrino and $\gamma$-ray) coincidence searches in near future. For variable sources, such as TXS 0506+056, we can use the muon neutrino fluence sensitivity, $\phi_{\text {lim }} \sim 0.04 \mathrm{GeV} \mathrm{cm}^{-2}$ (for a spectrum that is broad around $0.1 \mathrm{PeV}$ ), which can also be calculated from the public effective area. Due to the $\gamma$-ray monitoring of blazars with Fermi LAT in GeV energies and HAWC in TeV energies, the detection rate of flaring blazars in neutrinos can be estimated to be (see Eq. 4.14 of Murase et al. 2012a)

$$
\begin{aligned}
\dot{N}_{\text {blazar }} & \approx \frac{\Delta \Omega}{3} \rho_{0}^{\text {eff }}\left(\frac{\varepsilon_{\nu} L_{\varepsilon_{\nu \mu}}^{\mathrm{fl}} t_{\mathrm{dur}}}{4 \pi \phi_{\text {lim }}}\right)^{3 / 2} \\
& \lesssim 0.9 \mathrm{yr}^{-1}\left(\frac{f_{\mathrm{fl}}}{0.05}\right)^{-1 / 2} t_{\mathrm{dur}, 7}^{1 / 2}\left(\frac{F_{\text {lim },-9.2}}{\phi_{\text {lim },-1.4}}\right)^{3 / 2}
\end{aligned}
$$

Here $\rho_{0}^{\text {eff }}=f_{\text {fl }} n_{0}^{\text {eff }} / t_{\text {dur }}$ is the effective rate density of blazar flares, and the above equation is valid when the IceCube observation time is longer than $t_{\mathrm{dur}} / f_{\mathrm{fl}}$. This estimate is consistent not only with existing observational constraints but also with the fact that no other flares besides that of TXS $0506+056$ have been identified with a high significance. The prospects of detecting neutrinos from short-duration blazar flares are less favorable, because of $\dot{N}_{\text {blazar }} \propto t_{\text {dur }}^{1 / 2}$ (see also Petropoulou et al. 2016; Guépin \& Kotera 2017). If the association with TXS $0506+056$ is physical, according to the standard leptonic scenario including FSRQs (Murase et al. 2014; Dermer et al. 2014), we predict that neutrinos associated with FSRQ flares should also be identified in the future. It also suggests that dedicated time-dependent neutrino searches (Turley et al. 2016, 2018) are important to test these predictions.

\section{IMPLICATIONS FOR SOURCE MODELS}

\subsection{Importance of X-Ray and $\gamma$-Ray Observations}

Keivani et al. (2018) provided a detailed study of the TXS 0506+056 flare, using the multi-messenger data that have been obtained quasi-simultaneously with IceCube-170922A. The authors found a viable model for both high-energy neutrinos and $\gamma$-rays only in the leptonic scenario, where $\gamma$-ray emission is attributed to the inverse Compton (IC) mechanism. The X-ray and $\gamma$-ray light curves were variable on a day timescale, thus implying a comoving size of the emission region of $l^{\prime} \approx \delta c t_{\mathrm{var}} /(1+z) \simeq 4.5 \times 10^{16} \mathrm{~cm}(\delta / 20) t_{\mathrm{var}, 5}$, where typical values of the Doppler factor are $\delta \sim 10-30$. The observed SED suggests that the Compton dominance parameter is around unity, suggesting a magnetic field of $B^{\prime} \sim 0.1-1 \mathrm{G}$ (Keivani et al. 2018), which is consistent with population-based estimates for BL Lacs (Celotti \& Ghisellini 2008; Murase et al. 2014).

Neutrinos and hadronic $\gamma$-rays are coproduced by photomeson production, which is characterized by its effective optical depth, $f_{p \gamma}$. Let us consider a relativistically moving blob and a target photon spectrum, $n_{\varepsilon_{t}^{\prime}}$ (where $\varepsilon_{t}^{\prime} \approx \varepsilon_{t} / \delta$ is the target photon energy in the comoving frame). Approximating the spectrum by $\varepsilon^{\prime}{ }_{t} n_{\varepsilon_{t}^{\prime}}=n_{0}^{\prime}\left(\varepsilon^{\prime}{ }_{t} / \varepsilon^{\prime}{ }_{0}\right)^{1-\beta}$ with $\beta>1$, where $\varepsilon^{\prime}{ }_{0}$ is the reference energy, $f_{p \gamma}$ is given by (e.g., Murase et al. 2016)

$$
f_{p \gamma}\left[\varepsilon_{p}\right] \approx \eta_{p \gamma}[\beta] \hat{\sigma}_{p \gamma} l^{\prime} n_{0}^{\prime}\left(\varepsilon_{p}^{\prime} / \tilde{\varepsilon}_{p \gamma 0}^{\prime}\right)^{\beta-1},
$$

where $\eta_{p \gamma}[\beta] \approx 2 /(1+\beta), \hat{\sigma}_{p \gamma} \sim 0.7 \times 10^{-28} \mathrm{~cm}^{2}$ is the attenuation cross section, $\bar{\varepsilon}_{\Delta} \sim 0.3 \mathrm{GeV}$, and $\tilde{\varepsilon}_{p \gamma 0}^{\prime}=0.5 m_{p} c^{2} \bar{\varepsilon}_{\Delta} / \varepsilon_{0}^{\prime}$. This estimate is valid when the meson production is dominated by the $\Delta$-resonance and direct pion production. For target photons with observed energy $E_{t}=\varepsilon_{t} /(1+z)$, the characteristic energy of protons producing neutrinos of energy $\varepsilon_{\nu}$ is $\varepsilon_{p} \approx 20 \varepsilon_{\nu} \approx 0.5 \delta^{2} m_{p} c^{2} \bar{\varepsilon}_{\Delta} \varepsilon_{t}{ }^{-1}$. This results in $\varepsilon_{t} \sim 8 \mathrm{keV}(\delta / 20)^{2}\left(\varepsilon_{\nu} / 300 \mathrm{TeV}\right)^{-1}$, corresponding to $\mathrm{UV}$ photons or X-rays for neutrino energies ranging from $\sim 3 \mathrm{PeV}$ to $\sim 30 \mathrm{TeV}$. For example, the highenergy synchrotron component of the SED during the TXS $0506+056$ flare is well described by $\beta=2.8$ in the optical-to-X-ray range (above the lower-energy peak at $\varepsilon_{\text {syn }} \sim 1 \mathrm{eV}$ ) (Keivani et al. 2018). In this case, for a CR spectrum with $s_{\text {cr }}=2$, the predicted neutrino spectrum is so hard (see also Eq. 9) that it would contradict the nondetection of $>10 \mathrm{PeV}$ neutrinos during the flare, unless the CR proton spectrum cuts off at $10-100 \mathrm{PeV}$ (Keivani et al. 2018).

The same target photons lead to the Bethe-Heitler 
pair production, to which the effective optical depth is:

$$
\begin{aligned}
f_{\mathrm{BH}}\left[\varepsilon_{p}\right] & \approx \eta_{p \gamma}[\beta] \hat{\sigma}_{\mathrm{BH}} l^{\prime} n_{0}^{\prime}\left(\varepsilon_{p}^{\prime} / \tilde{\varepsilon}_{\mathrm{BHO}}^{\prime}\right)^{\beta-1} \\
& =g[\beta] f_{p \gamma}\left[\varepsilon_{p}\right],
\end{aligned}
$$

where $\hat{\sigma}_{\mathrm{BH}} \sim 0.8 \times 10^{-30} \mathrm{~cm}^{2}, \quad \bar{\varepsilon}_{\mathrm{BH}} \sim$ $10\left(2 m_{e} c^{2}\right) \sim 10 \mathrm{MeV}$ (Chodorowski et al. 1992), $\tilde{\varepsilon}_{\mathrm{BH} 0}^{\prime}=0.5 m_{p} c^{2} \bar{\varepsilon}_{\mathrm{BH}} / \varepsilon_{0}^{\prime}$, and $g[\beta] \sim 0.011(30)^{\beta-1}$.

The same photons also prevent $\gamma$-rays from escaping the source. The $\gamma \gamma$ optical depth can be written in terms of $\varepsilon_{\nu}$ (see Eq. 8 in Murase et al. 2016) as:

$$
\tau_{\gamma \gamma}\left[\varepsilon_{\gamma \gamma-p \gamma}\right] \sim 10^{3} f_{p \gamma}\left[20 \varepsilon_{\nu}\right],
$$

where $\varepsilon_{\gamma \gamma-p \gamma} \sim 10 \mathrm{GeV}\left(\varepsilon_{\nu} / 300 \mathrm{TeV}\right)$. The fact that $10-100 \mathrm{GeV} \gamma$-rays were observed during the flare suggests that the neutrino production in the same emission region has to be inefficient (e.g., Waxman \& Bahcall 1997; Levinson 2006; Dermer et al. 2007; Murase et al. 2016; Petropoulou et al. 2017). Imposing $\tau_{\gamma \gamma}<1$ at $100 \mathrm{GeV}$ leads to $f_{p \gamma}<10^{-3}\left(\varepsilon_{p} / 60 \mathrm{PeV}\right)^{\beta-1}$. Note that the neutrino energy is related to the proton energy as $\varepsilon_{\nu} \approx 0.05 \varepsilon_{p}=0.3 \mathrm{PeV}\left(\varepsilon_{p} / 6 \mathrm{PeV}\right)$.

For an isotropic-equivalent proton luminosity, $\varepsilon_{p} L_{\varepsilon_{p}}$, the differential neutrino luminosity is then given by

$$
\begin{aligned}
\varepsilon_{\nu} L_{\varepsilon_{\nu}} & \approx \frac{3}{8} f_{p \gamma}\left(\varepsilon_{p} L_{\varepsilon_{p}}\right) \\
& \simeq 1.2 \times 10^{45} \mathrm{erg} \mathrm{s}^{-1} f_{p \gamma,-4}\left(\frac{\varepsilon_{p} L_{\varepsilon_{p}}}{10^{49.5} \mathrm{erg} \mathrm{s}^{-1}}\right)
\end{aligned}
$$

which is consistent with the results of Keivani et al. (2018). The remaining fraction (i.e., 5/8) of energy should be carried by pionic $\gamma$-rays with $\varepsilon_{\gamma}^{\prime} \approx$ $0.1 \varepsilon_{p}^{\prime}$ and secondary electrons and positrons with $\gamma_{e}^{\prime} \approx$ $0.05 \varepsilon_{p}^{\prime} /\left(m_{e} c^{2}\right) \simeq 2.9 \times 10^{7}\left(\varepsilon_{p} / 6 \mathrm{PeV}\right)(20 / \delta)$. The TeV$\mathrm{PeV} \gamma$-rays are attenuated inside the source, which also generate the pairs. The Bethe-Heitler process also injects high-energy pairs with $\gamma_{e}^{\prime} \approx 5 \times 10^{-4} \varepsilon_{p}^{\prime} /\left(m_{e} c^{2}\right) \simeq$ $2.9 \times 10^{5}\left(\varepsilon_{p} / 6 \mathrm{PeV}\right)(20 / \delta)$ (e.g. Mastichiadis \& Kirk 1995); even more energetic pairs can be produced by interactions happening far from the energy threshold of the process (e.g. Kelner \& Aharonian 2008). These highly relativistic pairs quickly lose their energies via synchrotron and IC cooling. The cooling Lorentz factor is $\gamma_{c}^{\prime} \approx 2300 B_{-0.5}^{-2} l_{17}^{\prime-1}\left(1+Y_{\mathrm{IC}}\right)^{-1}$, implying that the resulting cascade spectrum lies in the fast-cooling regime. In the case of TXS 0506+056, the synchrotron peak is comparable to the IC peak, and the Compton Y parameter $\left(Y_{\mathrm{IC}}\right)$ is at most unity (Keivani et al. 2018).

The synchrotron emission from pairs injected via the Bethe-Heitler process is not always negligible in blazars, as demonstrated by Petropoulou \& Mastichiadis (2015). It turns out to be important also for TXS 0506+056 during its high state (Keivani et al. 2018). The minimum synchrotron cascade flux associated with the neutrino flux at $\varepsilon_{\nu}$ is:

$$
\begin{aligned}
\left.\varepsilon_{\gamma} L_{\varepsilon_{\gamma}}\right|_{\varepsilon_{\mathrm{syn}}^{\mathrm{BH}}} & \approx \frac{1}{2\left(1+Y_{\mathrm{IC}}\right)} g[\beta] f_{p \gamma}\left(\varepsilon_{p} L_{\varepsilon_{p}}\right) \\
& \approx \frac{4 g[\beta]}{3\left(1+Y_{\mathrm{IC}}\right)} \varepsilon_{\nu} L_{\varepsilon_{\nu}},
\end{aligned}
$$

where $\varepsilon_{\mathrm{syn}}^{\mathrm{BH}} \simeq 6 \mathrm{keV} B^{\prime}{ }_{-0.5}\left(\varepsilon_{p} / 6 \mathrm{PeV}\right)^{2}(20 / \delta)$ is the characteristic frequency of synchrotron emission by pairs from protons with $\varepsilon_{p} \approx 20 \varepsilon_{\nu}$. Because of the broad distribution of pairs injected by the Bethe-Heitler processes, even if the protons are monoenergetic, (Dimitrakoudis et al. 2012) the expected synchrotron spectrum will be extending over several decades in energy (e.g. Petropoulou \& Mastichiadis 2015; Petropoulou et al. 2015). Note that for sufficiently high-energy pairs we expect $Y_{\text {IC }} \ll 1$ due to the KleinNishina suppression.

Similarly, for synchrotron emission from pairs injected via photomeson production and two-photon annihilation for pionic $\gamma$-rays, the synchrotron cascade flux is:

$$
\begin{aligned}
\left.\varepsilon_{\gamma} L_{\varepsilon_{\gamma}}\right|_{\varepsilon_{\mathrm{syn}}^{p \gamma}} & \approx \frac{1}{2\left(1+Y_{\mathrm{IC}}\right)} \frac{5}{8} f_{p \gamma}\left(\varepsilon_{p} L_{\varepsilon_{p}}\right) \\
& \approx \frac{5}{6\left(1+Y_{\mathrm{IC}}\right)} \varepsilon_{\nu} L_{\varepsilon_{\nu}},
\end{aligned}
$$

where $\varepsilon_{\mathrm{syn}}^{p \gamma} \simeq 60 \mathrm{MeV} B^{\prime}{ }_{-0.5}\left(\varepsilon_{p} / 6 \mathrm{PeV}\right)^{2}(20 / \delta)$ and the contribution of pionic $\gamma$-rays is included assuming that they are converted into pairs inside the source.

In addition to the synchrotron cascade components considered above, the IC emission and subsequent regeneration processes can affect the pair-injection spectrum. Although the exact spectral shape of a cascade photon spectrum depends on details of the pair injection and possible contributions from muon and meson radiation, the resulting energy spectrum becomes approximately flat, that is, it can be expressed as $E_{\gamma} F_{E_{\gamma}} \propto E_{\gamma}^{2-\beta}$ with $\beta \sim 1.5-2$ varying in the $\mathrm{X}$-ray and $\gamma$-ray range.

For the TXS 0506+056 flare coincident with IceCube170922A, Swift and NuSTAR measured X-rays quasisimultaneously. A more recent sophisticated analysis gave the X-ray flux, $E_{\gamma} F_{E_{\gamma}}^{X} \approx 0.8 \times 10^{-12} \mathrm{erg} \mathrm{cm}^{-2} \mathrm{~s}^{-1}$ at $E_{\gamma} \approx 2-3 \mathrm{keV}$ (Keivani et al. 2018). This leads to tight limits on the high-energy neutrino flux from the TXS 0506+056 flare. Combining Eq. (13) with the observed X-ray flux, the neutrino flux in the $0.1-1 \mathrm{PeV}$ range is constrained as $E_{\nu} F_{E_{\nu}}^{0.1-1} \mathrm{PeV} \lesssim$ $E_{\gamma} F_{E_{\gamma}}^{X} \sim 10^{-12} \mathrm{erg} \mathrm{cm}^{-2} \mathrm{~s}^{-1}$ for all flavors, or we have $\varepsilon_{\nu} L_{\varepsilon_{\nu_{\mu}}}^{0.1-1} \mathrm{PeV} \lesssim \varepsilon_{\gamma} L_{\varepsilon_{\gamma}}^{X} / 3 \sim 10^{44} \mathrm{erg} \mathrm{s}^{-1}$, where a factor of 3 comes from $\nu_{e}: \nu_{\mu}: \nu_{\tau} \approx 1: 1: 1$. This is fully consistent with the detailed numerical results presented in Keivani et al. (2018), and the upper limit neutrino luminosity is much lower than the luminosity suggested for the 2017 and 2014-2015 flares of TXS 0506+056, $\varepsilon_{\nu} L_{\varepsilon_{\nu}}\left(\approx 3 \varepsilon_{\nu} L_{\varepsilon_{\nu_{\mu}}}\right) \sim 10^{46}-10^{47} \mathrm{erg} \mathrm{s}^{-1}$. Note that 
the neutrino luminosity around the peak at higher energies (see Fig. 4 of Keivani et al. 2018) is slightly higher owing to $g[\beta]$ and details of the cascade spectrum.

Eqs. (13) and (14) show that the luminosity of the synchrotron cascade components is comparable to the neutrino luminosity, as long as $Y_{\mathrm{IC}} \ll 1$. Thus, the cascade bound on the neutrino flux is unavoidable as long as the photomeson production occurs in a compact region such as the blazar zone. If the canonical picture of blazars based on the single-zone modeling is correct, these results allow us to predict that the 2017 and 20142015 neutrino flares reported by the IceCube Collaboration (IceCube-Collaboration 2018) should be accompanied by X-ray emission with $E_{\gamma} F_{E_{\gamma}}^{X} \sim E_{\nu} F_{E_{\nu}}^{0.1-1} \mathrm{PeV} \sim$ $(3-30) \times 10^{-11} \mathrm{erg} \mathrm{cm}^{-2} \mathrm{~s}^{-1}$, which should be detectable by X-ray sky monitors such as Swift and MAXI.

Finally, we comment on high-energy neutrino emission through hadronuclear processes. For a relativistic jet of blazars, $p p$ interactions as the neutrino production mechanism cannot be globally efficient at least in the blazar zone; otherwise the jet becomes too heavy to remain relativistic. However, for flares, one could potentially avoid this problem by invoking some mechanism to entrain dense clouds in the jets. Several possibilities have been discussed, which include clouds in the broad-line region, stellar winds, and the tidal stripping of the stellar envelope (e.g., Bednarek \& Protheroe 1997; Bosch-Ramon et al. 2012; Perucho et al. 2017). In addition, massive stars could supply matter via supernova explosions or mass eruptions. Although various possibilities could potentially be considered (see Fig. 2), there are several issues in the explanation for the month-scale neutrino flares from TXS $0506+056$. First, there has been much evidence that the clouds in the broad-line region are mostly located around the equatorial plane, not on the jet axis (e.g., Wills \& Browne 1986; Runnoe et al. 2013; Shen \& Ho 2014). The profiles of the broad-line emission often show a disk-like rotation (e.g., Eracleous et al. 2009; Luo et al. 2013), and the vertical velocity component can be naturally explained by the dusty disk wind (e.g., Czerny \& Hryniewicz 2011; Czerny et al. 2015). On the other hand, there are a large number of stars around the AGN core, and some of them could be on the jet axis. No matter what the origin is, the cloud mass required to achieve a high column density of $\gtrsim 10^{24} \mathrm{~cm}^{-2}$ is $M_{c} \gtrsim 0.4 M_{\odot} l_{c, 16}^{2}$ for the cloud size $l_{c}$ (note that the observations suggest that the comoving size of the blazar zone is $\left.l^{\prime} \sim 10^{16}-10^{17} \mathrm{~cm}\right)$. With a typical jet luminosity of BL Lacs, it is difficult for a massive cloud to become highly relativistic. If the $\mathrm{CR}$ generation occurs via collisions between the jet and a dense cloud at rest, the characteristic time scale is given by $(1+z) l_{c} / \delta^{2} c \simeq 1.1 \times 10^{4} \mathrm{~s} l_{c, 17}(20 / \delta)^{2}$, which is shorter than the observed variability and duration, although the evaporation and expansion subsequent to the jet-cloud interaction may eventually lead to a longer time scale. Third, the existence of a powerful jet implies that such a cloud can be largely ionized and strong photoelectric absorption of X-rays is unlikely to happen except for the innermost region of the cloud. For a cloud density of $n_{c} \simeq 2.8 \times 10^{6} \mathrm{~cm}^{-3}\left(M_{c} / 10 M_{\odot}\right) l_{c, 17}^{-3}$, the ionization radius is estimated to be $\sim 8 \times$ $10^{17} \mathrm{~cm}\left(\varepsilon_{\gamma} L_{\varepsilon_{\gamma}} / 10^{45} \mathrm{erg} \mathrm{s}^{-1}\right)^{1 / 3}\left(\varepsilon_{\gamma} / 100 \mathrm{eV}\right)^{-1 / 3} n_{c, 7}^{-2 / 3} \mathcal{T}_{5}^{1 / 4}$, where $\mathcal{T}$ is the cloud temperature. Except for extreme cases with $N_{H} \gg \sigma_{T}^{-1}$, we expect that X-rays escape and the cascade limit is important even for $p p$ models, and we have:

$$
\begin{aligned}
\left.\varepsilon_{\gamma} L_{\varepsilon_{\gamma}}\right|_{\varepsilon_{\text {syn }}^{p p}} & \approx \frac{1}{2\left(1+Y_{\mathrm{IC}}\right)} \frac{1}{2} f_{p p}\left(\varepsilon_{p} L_{\varepsilon_{p}}\right) \\
& \approx \frac{1}{2\left(1+Y_{\mathrm{IC}}\right)} \varepsilon_{\nu} L_{\varepsilon_{\nu}} .
\end{aligned}
$$

For a spectrum of CRs with $s_{\text {cr }} \sim 2$, the above relation holds at energies from $\varepsilon_{\mathrm{syn}}^{c} \simeq$ $0.39 \mathrm{eV} B_{-0.5}^{\prime-3} l_{17}^{\prime-2}\left(1+Y_{\mathrm{IC}}\right)^{-2}(\delta / 20)$ to $\varepsilon_{\mathrm{syn}}^{p p \max } \simeq$ $60 \mathrm{MeV} B^{\prime}{ }_{-0.5}\left(\varepsilon_{p}^{\max } / 6 \mathrm{PeV}\right)^{2}(20 / \delta)$.

\subsection{Multizone Models and CR-induced Neutral Beams}

An electromagnetic cascade is a consequence of energy conservation, so the cascade limit discussed in the previous subsection exists for not only single-zone but also multizone models.

One of the possibilities is that neutrinos are mainly produced around the base of the jet or even in the vicinity of a supermassive black hole (i.e., AGN core models) (e.g., Stecker et al. 1991; Bednarek \& Protheroe 1999; Becker \& Biermann 2009; Stecker 2013; Kimura et al. 2015). This includes highenergy neutrino production caused by $p p$ interactions with dense clouds as discussed above. Because of the higher compactness of the system, broadband cascade emission should accompany the neutrino emission. In such an inner region, magnetic fields should also be much stronger, which could affect the resulting cascade spectrum. Importantly, not only protons but also electrons are accelerated, and both emission components should be taken into account. Detailed studies are beyond the scope of this work (Mastichiadis \& Petropoulou 2018 in preparation).

Here we consider the CR-induced neutral beam model (see Fig. 2), which can avoid the cascade constraints. We consider interactions between beamed CRs escaping from the blazar zone and an external radiation field. Although we do not specify the origin of the external photons, this setup is analogous to the one considered in Murase et al. (2014) and Dermer et al. (2014). In this sense, this two-zone model can be regarded as a natural extension of the standard leptonic scenario. In particular, we assume that escaping CRs are neutrons 
that can be produced via the photodisintegration of nuclei in the blazar zone. The $\gamma$-ray signatures produced by CR-induced neutral beams were previously studied in Murase (2012) and Dermer et al. (2012) (see also Essey et al. 2010, 2011; Murase et al. 2012b, for related discussion about intergalactic cascades). As we show below, the cascade emission can be largely diminished via the isotropization of relativistic electrons and positrons.

Following Dermer et al. (2014), let us assume that CRs are accelerated via the second-order Fermi acceleration mechanism. The maximum energy accelerated in the blazar zone can be $\varepsilon_{\mathrm{cr}} / Z \sim 1-10 \mathrm{PeV}$, where $\varepsilon_{\mathrm{cr}}$ is the CR ion energy and $Z$ is the nuclear charge. The $\mathrm{CR}$ acceleration zone can be the $\gamma$-ray emission site or inner regions of the blazar zone, and disintegrated nuclei are accompanied by not only protons but also neutrons (e.g., Murase \& Beacom 2010; Rodrigues et al. 2018). The protons may lose energy via adiabatic losses during the confinement in the blazar zone, while neutrons can escape. The neutron luminosity is given by $\varepsilon_{n} L_{\varepsilon_{n}} \approx(1 / 2) f_{A \gamma}\left(\varepsilon_{\mathrm{cr}} L_{\varepsilon_{\mathrm{cr}}}\right)$, where $f_{A \gamma}$ is the effective optical depth to the photodisintegration process (Murase \& Beacom 2010) and $\varepsilon_{\mathrm{cr}} L_{\varepsilon_{\mathrm{cr}}}$ is the CR ion luminosity. For neutron production in the $\gamma$-ray emission region, we have $f_{A \gamma} \sim$ $0.1\left(\varepsilon_{\gamma} L_{\varepsilon_{\gamma}} / 10^{46} \mathrm{erg} \mathrm{s}^{-1}\right)(\delta / 20)^{-3} l_{17}^{\prime-1}\left(\varepsilon_{\gamma} / 1 \mathrm{eV}\right)^{-1}$ $\left(\varepsilon_{\mathrm{cr}}^{\prime} / \tilde{\varepsilon}_{A \gamma, \mathrm{syn}}^{\prime}\right)^{\beta-1}$, where $\tilde{\varepsilon}_{A \gamma, \mathrm{syn}}^{\prime}=0.5 m_{A} c^{2} \bar{\varepsilon}_{\mathrm{GDR}} / \varepsilon_{\mathrm{syn}}^{\prime}$ and $\bar{\varepsilon}_{\mathrm{GDR}} \sim 20-30 \mathrm{MeV}$. This also implies that the neutron emission may predominantly come from smaller dissipation radii at which efficient photodisintegration (i.e., $f_{A \gamma} \gtrsim 1$ ) occurs.

In single-zone models, the neutrino flares of TXS 0506+056 require unpleasantly large CR luminosities (Keivani et al. 2018). This problem still exists at some level even in multizone models, although it can be alleviated in the $\mathrm{CR}$ beam model in the sense that the meson production efficiency is enhanced by additional target photons or nucleons. One should keep in mind that observations and modeling of radio galaxies (based on larger-scale jets than the blazar zone) have shown that the absolute jet power averaged over the lifetime of the AGN jet is $P_{j} \lesssim 10^{45}-10^{46} \mathrm{erg} \mathrm{s}^{-1}$ for Fanaroff-Riley I galaxies (Cavagnolo et al. 2010; Godfrey \& Shabala 2013), which are are believed to be off-axis counterparts of BL Lacs. For the supermassive black hole mass $M_{\mathrm{BH}}$, the Eddington luminosity ${ }^{3}$ is $L_{\mathrm{Edd}} \simeq 1.3 \times$ $10^{47} \mathrm{erg} \mathrm{s}^{-1}\left(M_{\mathrm{BH}} / 10^{9} M_{\odot}\right)$. Ghisellini et al. (2014) showed that the absolute jet power of blazars may exceed the accretion luminosity, and our study implies

3 The X-ray observations (Keivani et al. 2018) indicate that the disk luminosity in the X-ray range has to be lower than $3 \times 10^{44} \mathrm{erg} \mathrm{s}^{-1}$, which is consistent with the common belief that BL Lacs are associated with radiatively inefficient accretion disks.

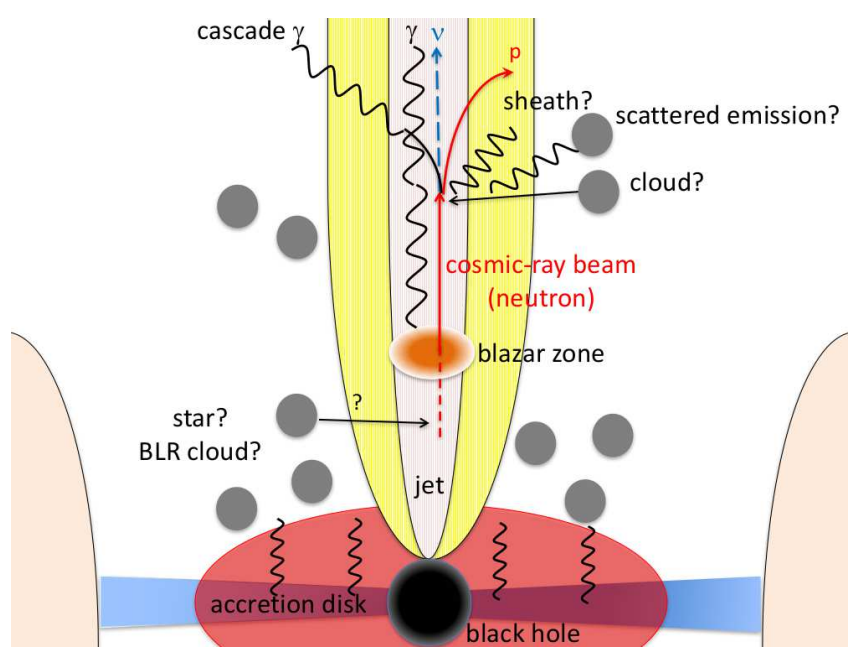

Figure 2. Schematic picture (not in scale) of the CR-induced beam model for high-energy neutrino production. See text for details (see also Murase et al. 2014; Dermer et al. 2012). While the neutrino emission is highly beamed, the associated cascade emission in the X-ray range is isotropized.

that the flaring jet power is larger than the timeaveraged one by $b_{\mathrm{fl}} / f_{\mathrm{fl}} \sim 3-10$. The isotropicequivalent $\mathrm{CR}$ luminosity during the flaring phase can then be written as $L_{\mathrm{cr}}^{\mathrm{fl}} \approx\left(2 / \theta_{\text {beam }}^{2}\right) \epsilon_{\mathrm{cr}} P_{j}\left(b_{\mathrm{fl}} / f_{\mathrm{fl}}\right) \simeq 6.0 \times$ $10^{49} \mathrm{erg} \mathrm{s}^{-1}\left(\theta_{\text {beam }} / 0.05\right)^{-2}\left(\epsilon_{\mathrm{cr}} / 0.2\right)\left(b_{\mathrm{ff}} f_{\mathrm{fl}}^{-1} / 10\right)$

$\left(P_{j} / 0.3 L_{\mathrm{Edd}}\right)\left(M_{\mathrm{BH}} / 10^{9} M_{\odot}\right)$, where $\epsilon_{\mathrm{cr}}$ is the energy fraction carried by $\mathrm{CR}$ ions and $\theta_{\text {bream }}$ is the opening angle of the CR beam. The neutron luminosity during the flaring phase results in $L_{n}^{\mathrm{fl}} \simeq 3.0 \times$ $10^{49} \mathrm{erg} \mathrm{s}^{-1} f_{A \gamma}\left(\theta_{\text {beam }} / 0.05\right)^{-2}\left(\epsilon_{\mathrm{cr}} / 0.2\right)\left(b_{\mathrm{fl}} f_{\mathrm{fl}}^{-1} / 10\right)$ $\left(P_{j} / 0.3 L_{\mathrm{Edd}}\right)\left(M_{\mathrm{BH}} / 10^{9} M_{\odot}\right)$.

The neutrons that leave the $\mathrm{CR}$ acceleration zone propagate along the jet and may interact with external radiation fields that could exist on larger scales or perhaps a dense cloud. For LSP and ISP objects like TXS $0506+056$, it is possible to invoke such a setup. For example, if the jet is structured, nonthermal photons can be provided by the sheath region. Moreover, a fraction of UV and X-ray emission from the accretion disk can be scattered by clumps of matter that may be present at outer radii. In addition, there could be highvelocity clumps such as the broad-line region, although they are usually seen in FSRQs. Note that the neutrons with $\gamma_{n} \sim 10^{7}-10^{8}$ can travel $\sim 0.1-1 \mathrm{kpc}$.

Interestingly, the detailed modeling of the SED of TXS 0506+056 (Keivani et al. 2018) already suggested that such an external radiation field is necessary to explain the $\mathrm{X}$-ray and $\gamma$-ray spectrum. If this is the case, it is natural for escaping CRs to keep interacting with the ambient photons, leading to the production of more neutrinos.

As a toy model, we assume that the decelerated jet or slower jet of the sheath region provides soft photons with a luminosity of $L_{\text {ext }} \sim 3 \times 10^{45} \mathrm{erg} \mathrm{s}^{-1}$ and the characteristic energy at $\varepsilon_{\text {ext }} \sim 10 \mathrm{eV}$, over 
a length scale of $R_{\text {ext }} \sim 3 \times 10^{19} \mathrm{~cm}$. The external radiation energy density is $U_{\text {ext }} \approx 3 L_{\text {ext }} /\left(4 \pi R_{\text {ext }}^{2} c\right) \sim$ $3 \times 10^{-5} \mathrm{erg} \mathrm{cm}^{-3}$, which is consistent with the parameters used in Keivani et al. (2018). Noting $\hat{\sigma}_{n \gamma} \approx$ $\hat{\sigma}_{p \gamma}$, the photomeson production efficiency is $f_{n \gamma} \approx$ $\left[\eta_{n \gamma} \hat{\sigma}_{n \gamma} 3 L_{\text {ext }} /\left(4 \pi R_{\text {ext }} c \varepsilon_{\text {ext }}\right)\right]\left(\varepsilon_{n} / \tilde{\varepsilon}_{n \gamma, \text { ext }}\right)^{\beta-1} \sim 3 \times$ $10^{-3} \eta_{n \gamma} L_{\text {ext }, 45.5} R_{\text {ext }, 19.5}^{-1}\left(\varepsilon_{\text {ext }} / 10 \mathrm{eV}\right)^{-1}\left(\varepsilon_{n} / \tilde{\varepsilon}_{n \gamma, \text { ext }}\right)^{\beta-1}$ (where $\eta_{n \gamma}=\eta_{p \gamma}$ and $\tilde{\varepsilon}_{n \gamma \text {,ext }}=0.5 m_{n} c^{2} \bar{\varepsilon}_{\Delta} / \varepsilon_{\text {ext }} \approx$ $\left.\tilde{\varepsilon}_{p \gamma, \text { ext }}\right)$, which can be larger by a factor of $\sim R_{\text {ext }} /\left(\Gamma l^{\prime}\right)$ compared to $f_{p \gamma}$ in the blazar zone (see Eq. 9). Alternatively, instead of $n \gamma$ interactions, one could expect $n p$ interactions between the neutral beam and a dense cloud in the line of sight. Although the existence of such a cloud is speculative, with a clump like a giant molecular cloud with a mass of $M_{c} \sim\left(10^{5}-10^{6}\right) M_{\odot}$ and a size of $l_{c} \sim 10^{19} \mathrm{~cm}$, the effective $n p$ optical depth can be as high as $f_{n p} \simeq 0.011\left(M_{\mathrm{c}} / 10^{5} M_{\odot}\right) l_{c, 19}^{-2}$.

The all-flavor neutrino luminosity is:

$\varepsilon_{\nu} L_{\varepsilon_{\nu}} \simeq 3.8 \times 10^{46} \mathrm{erg} \mathrm{s}^{-1}\left(\frac{2 K}{1+K}\right)\left(\frac{f_{n \gamma / n p} \varepsilon_{n} L_{\varepsilon_{n}}}{10^{47} \mathrm{erg} \mathrm{s}^{-1}}\right)$,

where $K=1$ and $K=2$ for $n p$ and $n \gamma$ interactions, respectively. In the $n \gamma$ case, the neutrino energy corresponding to the neutron energy with $\tilde{\varepsilon}_{n \gamma \text {,ext }}$ is:

$$
\varepsilon_{\nu} \approx 0.05 \tilde{\varepsilon}_{n \gamma, \text { ext }} \sim 0.7 \mathrm{PeV}\left(\varepsilon_{\text {ext }} / 10 \mathrm{eV}\right)^{-1},
$$

which could explain both of the 2017 and 2014-2015 neutrino flares of TXS 0506+056, e.g., with $f_{A \gamma} \sim 0.1$ and $f_{A \gamma} \gtrsim 1$, respectively. The duration of neutrino emission is comparable to the "lifetime" of the CR-induced beam, which is being determined by the duration of particle energization in the $\mathrm{CR}$ acceleration zone corresponding to the observed $t_{\text {dur }}$.

The key point of the CR-induced neutral beam model considered here is that the cascade signature except for $\gamma$-rays in the very high-energy range can be largely diminished because of the isotropization of the relativistic pairs in the larger-scale jet or other magnetized environments. Let us assume that the magnetic field in the main scale of neutrino production is as small as $B_{\text {ext }} \sim 0.1-10 \mathrm{mG}$ (typical for large-scale jets). The deflection of pairs occurs before they cool via synchrotron and IC losses. Following Murase (2012), the deflection angle during the radiation cooling time is given by:

$$
\theta_{\mathrm{def}} \approx \sqrt{\frac{2}{3}} \frac{c t_{\mathrm{syn}}}{r_{L}} \simeq 3.5 \gamma_{e, 9}^{-2} B_{\mathrm{ext},-2.5}^{-1},
$$

where $t_{\mathrm{syn}}$ is the synchrotron cooling time, $r_{L}$ is the Larmor radius, and the Lorentz factor of pairs in the black hole rest frame is $\gamma_{e} \approx 0.05 \varepsilon_{n} /\left(m_{e} c^{2}\right) \simeq 5.8 \times$ $10^{8}\left(\varepsilon_{n} / 6 \mathrm{PeV}\right)$. Only synchrotron cooling is considered, because the IC cooling is suppressed for such highenergy electrons and positrons. The above equation implies that the pairs lose their energy after they become isotropized. Note that the X-ray emission is doubly sup- pressed, because the deflection is larger than the jet opening angle, i.e., $\theta_{\text {def }} \gg \theta_{j}$, and the time spread in the cascade emission is longer than the intrinsic flare duration, i.e., $R_{\text {ext }} / c \gg t_{\text {dur. }}$. Also, the CR neutrons do not produce extra pairs via the Bethe-Heitler process. In addition, in the $n p$ scenario, X-rays could also be isotropized via the Compton scattering. Thus, X-rays from the neutral beam can be highly suppressed if the CR energy is lower than UHE energies, and the associated hadronic cascade signatures can be masked by the $\mathrm{GeV} \gamma$-ray emission from the blazar zone. Note that the UHE neutron beam, if it exists, can also produce beamed cascade emission at $\gtrsim 0.1 \mathrm{TeV}$ energies. The resulting synchrotron pair-echo emission (Murase 2012; Dermer et al. 2012) is expected to have some time delay. We also remark that the very high-energy flare seen by MAGIC detected about 10 days later after IceCube170922A.

\section{SUMMARY}

We considered implications of the high-energy neutrino flares from TXS $0506+056$ by examining various constraints. Flaring blazars could be the brightest sources in the neutrino sky, while the observations can most naturally be reconciled with existing theoretical models, if the blazars are subdominant $(\sim 1-10 \%)$ in the diffuse neutrino intensity. Interestingly, within the standard leptonic scenario of $\gamma$-ray emission, we found that the blazar neutrino emission itself can readily be dominated by flaring episodes. This could explain why the significant neutrino signals from TXS 0506+056 were found only in the time-dependent search and that the steady component that typically dominates $\gamma$-ray emission has not been seen yet in neutrinos. Bright neutrino flares like the ones observed for TXS 0506+056 could contribute only up to a few percent of the INB. If the association with this blazar is physical, such flares can be detected with a rate of $\lesssim 1 \mathrm{yr}^{-1}$ by dedicated time-dependent searches in the near future.

Based on analytical considerations, we also showed the importance of X-ray constraints to test the physical models of TXS 0506+056. An efficient electromagnetic cascade is unavoidable in the canonical blazar models based on the single radiation zone, which also predicts that the 2014-2015 neutrino flare found in the archival data should be accompanied by X-ray emission with $E_{\gamma} F_{E_{\gamma}}^{X} \sim(3-30) \times 10^{-11} \mathrm{erg} \mathrm{cm}^{-2} \mathrm{~s}^{-1}$. This can readily be tested by X-ray sky monitors such as Swift and MAXI. Nondetection of the X-ray flares will necessitate more complicated models involving multizone emission. As a possible example, we discussed the CRinduced neutral beam model. In this model, neutrino production is expected to occur via, e.g., photomeson production on external radiation fields, which were also inferred by the detailed modeling of the SED of TXS 
0506+056 (Keivani et al. 2018). Remarkably, the cascade emission, which is unavoidable whether neutrinos are produced by either photohadronic or hadronuclear process, can be largely diminished by the isotropization in magnetized environments and the absence of the Bethe-Heitler process, so that the X-ray constraints can be satisfied.

The reported significance of the neutrino flare from TXS $0506+056,3 \sigma-4 \sigma$, is intriguing. However, the observed coincidence still lacks convincing explanations in view of the multi-messenger data. More observational and theoretical efforts are necessary to confirm whether flaring blazars are the sources of high-energy neutrinos.

Even if blazars are established as the sources of neutrinos by future observations, our results imply that it will not address the two most important questions, that is, which source class the main origin of high-energy cosmic neutrinos is, and where UHECRs come from. Other sources or populations (or perhaps different regions) are most likely to be responsible for the bulk of high-energy cosmic neutrinos and UHECRs. Thus, next generation neutrino detectors such as IceCube-Gen2 (Aartsen et al. 2014) and KM3Net (Adrian-Martinez et al. 2016) will be necessary to address these puzzles.

We thank Derek Fox, Kunihito Ioka, Albrecht Karle, Nobuyuki Kawai, Azadeh Keivani, Shigeo Kimura, Peter Mészáros, Masaru Shibata, Yutaro Tachibana, Michael Unger, and Shigeru Yoshida for useful discussions. K.M. also acknowledges Niel Brandt and Amir Levinson for the discussions about the geometry of broad-line regions and jet energetics, respectively. The work of K.M. is supported by the Alfred P. Sloan Foundation and NSF grant No. PHY-1620777. F.O. acknowledges support from the DFG through grant SFB1258 "Neutrinos and Dark Matter in Astro- and Particle Physics". M.P. acknowledges support from the Lyman Jr. Spitzer Postdoctoral Fellowship. This research was supported by the Munich Institute for Astro- and Particle Physics (MIAPP) of the DFG cluster of excellence "Origin and Structure of the Universe".

\section{REFERENCES}

Aartsen, M., et al. 2013a, Phys.Rev.Lett., 111, 021103

—. 2013b, Science, 342, 1242856

—. 2014, arXiv:1412.5106

Aartsen, M. G., et al. 2015, Astropart. Phys., 66, 39

—. 2016, Phys. Rev. Lett., 117, 241101, [Erratum: Phys. Rev.

Lett.119,no.25,259902(2017)

—. 2017a, arXiv:1710.01191

—. 2017b, arXiv: 1710.01179

—. 2017c, Astrophys. J., 835, 45

Abbott, B., et al. 2017a, Phys. Rev. Lett., 119, 161101

Abbott, B. P., et al. 2016, Phys. Rev. Lett., 116, 061102

-. 2017b, Astrophys. J., 848, L13

Abdollahi, S., Ackermann, M., Ajello, M., et al. 2017, ApJ , 846, 34

Acero, F., et al. 2015, Astrophys. J. Suppl., 218, 23

Adrian-Martinez, S., et al. 2016, J. Phys., G43, 084001

Ahlers, M., \& Halzen, F. 2014, Phys.Rev., D90, 043005

Ajello, M., Romani, R., Gasparrini, D., et al. 2014, Astrophys.J., 780, 73

Ando, S., Feyereisen, M. R., \& Fornasa, M. 2017, Phys. Rev., D95, 103003

Atoyan, A., \& Dermer, C. D. 2001, Phys.Rev.Lett., 87, 221102

Becker, J. K., \& Biermann, P. L. 2009, Astroparticle Physics, 31, 138

Bednarek, W., \& Protheroe, R. J. 1997, Mon. Not. Roy. Astron. Soc., 287, L9

Bednarek, W., \& Protheroe, R. J. 1999, MNRAS , 302, 373

Bosch-Ramon, V., Perucho, M., \& Barkov, M. V. 2012, Astron. Astrophys., 539, A69

Bustamante, M., Baerwald, P., Murase, K., \& Winter, W. 2015, Nature Communications, 6, 6783

Cavagnolo, K. W., McNamara, B. R., Nulsen, P. E. J., et al. 2010, ApJ , 720, 1066

Celotti, A., \& Ghisellini, G. 2008, MNRAS , 385, 283

Chodorowski, M. J., Zdziarski, A. A., \& Sikora, M. 1992, ApJ 400, 181

Czerny, B., \& Hryniewicz, K. 2011, A\&A , 525, L8
Czerny, B., Modzelewska, J., Petrogalli, F., et al. 2015, Advances in Space Research, 55, 1806

Dermer, C. D., Murase, K., \& Inoue, Y. 2014, JHEAp, 3-4, 29

Dermer, C. D., Murase, K., \& Takami, H. 2012, Astrophys. J., 755,147

Dermer, C. D., Ramirez-Ruiz, E., \& Le, T. 2007, ApJL , 664, L67

Dimitrakoudis, S., Mastichiadis, A., Protheroe, R. J., \& Reimer, A. 2012, A\&A , 546, A120

Distefano, C., Guetta, D., Waxman, E., \& Levinson, A. 2002, Astrophys. J., 575, 378

Eracleous, M., Lewis, K. T., \& Flohic, H. M. L. G. 2009, NewAR, 53, 133

Essey, W., Kalashev, O., Kusenko, A., \& Beacom, J. F. 2011, Astrophys. J., 731, 51

Essey, W., Kalashev, O. E., Kusenko, A., \& Beacom, J. F. 2010, Phys. Rev. Lett., 104, 141102

Gao, S., Pohl, M., \& Winter, W. 2017, Astrophys. J., 843, 109

Ghisellini, G., Tavecchio, F., Maraschi, L., Celotti, A., \& Sbarrato, T. 2014, Nature, 515, 376

Godfrey, L. E. H., \& Shabala, S. S. 2013, Astrophys. J., 767, 12

Guépin, C., \& Kotera, K. 2017, A\&A , 603, A76

Gupta, N., \& Zhang, B. 2007, Astropart.Phys., 27, 386

Halzen, F. 2016, Nature Phys., 13, 232

Halzen, F., \& Hooper, D. 2005, Astropart. Phys., 23, 537

IceCube-Collaboration. 2018, Science, 361, 147

IceCube-Collaboration, Fermi-LAT, MAGIC, et al. 2018, Science, 361, 146

Kadler, M., et al. 2016, Nature Phys., 12, 807

Keivani, A., et al. 2018, Astrophys. J., 864, 84

Kelner, S. R., \& Aharonian, F. A. 2008, Phys. Rev. D. , 78 034013

Kimura, S. S., Murase, K., \& Toma, K. 2015, Astrophys.J., 806, 159

Levinson, A. 2006, Int. J. Mod. Phys., A21, 6015

Levinson, A., \& Waxman, E. 2001, Phys. Rev. Lett., 87, 171101

Lipari, P. 2008, Phys. Rev., D78, 083011

Lucarelli, F., et al. 2017, Astrophys. J., 846, 121 
Luo, B., Brandt, W. N., Eracleous, M., et al. 2013, MNRAS , 429, 1479

Mastichiadis, A., \& Kirk, J. G. 1995, A\&A , 295, 613

Murase, K. 2007, Phys.Rev., D76, 123001

—. 2008, AIP Conf. Proc., 1065, 201

—. 2012, Astrophys. J., 745, L16

-. 2017, in Neutrino Astronomy (Edited by Gaisser, T. and Karle, A.), 15-31, arXiv:1511.01590

—. 2018, Phys. Rev., D97, 081301

Murase, K., \& Beacom, J. F. 2010, Phys. Rev., D82, 043008

Murase, K., Beacom, J. F., \& Takami, H. 2012a, JCAP, 1208, 030

Murase, K., Dermer, C. D., Takami, H., \& Migliori, G. 2012b, Astrophys. J., 749, 63

Murase, K., Guetta, D., \& Ahlers, M. 2016, Phys. Rev. Lett., 116, 071101

Murase, K., Inoue, Y., \& Dermer, C. D. 2014, Phys.Rev., D90, 023007

Murase, K., \& Ioka, K. 2013, Phys.Rev.Lett., 111, 121102

Murase, K., Ioka, K., Nagataki, S., \& Nakamura, T. 2006, Astrophys.J., 651, L5

Murase, K., \& Nagataki, S. 2006, Phys. Rev., D73, 063002

Murase, K., Thompson, T. A., Lacki, B. C., \& Beacom, J. F. 2011, Phys.Rev., D84, 043003

Murase, K., \& Waxman, E. 2016, Phys. Rev., D94, 103006

Neronov, A., Semikoz, D. V., \& Ptitsyna, K. 2017, Astron. Astrophys., 603, A135

Paiano, S., Falomo, R., Treves, A., \& Scarpa, R. 2018, Astrophys. J., 854, L32

Palladino, A., Rodrigues, X., \& Winter, W. 2018, arXiv: 1806.04769

Palladino, A., \& Vissani, F. 2017, Astron. Astrophys., 604, A18

Perucho, M., Bosch-Ramon, V., \& Barkov, M. V. 2017, Astron. Astrophys., 606, A40

Petropoulou, M., Coenders, S., \& Dimitrakoudis, S. 2016, Astropart. Phys., 80, 115

Petropoulou, M., Coenders, S., Vasilopoulos, G., Kamble, A., \& Sironi, L. 2017, Mon. Not. Roy. Astron. Soc., 470, 1881

Petropoulou, M., Dimitrakoudis, S., Padovani, P., Mastichiadis, A., \& Resconi, E. 2015, Mon. Not. Roy. Astron. Soc., 448, 2412
Petropoulou, M., Giannios, D., \& Dimitrakoudis, S. 2014, Mon. Not. Roy. Astron. Soc., 445, 570

Petropoulou, M., \& Mastichiadis, A. 2015, MNRAS , 447, 36

Petropoulou, M., Nalewajko, K., Hayashida, M., \& Mastichiadis, A. 2017, MNRAS , 467, L16

Razzaque, S. 2013, Phys.Rev., D88, 103003

Resconi, E., Franco, D., Gross, A., Costamante, L., \& Flaccomio, E. 2009, A\&A , 502, 499

Rodrigues, X., Fedynitch, A., Gao, S., Boncioli, D., \& Winter, W. 2018, Astrophys. J., 854, 54

Runnoe, J. C., Brotherton, M. S., Shang, Z., Wills, B. J., \& DiPompeo, M. A. 2013, MNRAS , 429, 135

Shen, Y., \& Ho, L. C. 2014, Nat , 513, 210

Silvestri, A., \& Barwick, S. W. 2010, Phys.Rev., D81, 023001

Stecker, F. W. 2013, Phys.Rev., D88, 047301

Stecker, F. W., Done, C., Salamon, M. H., \& Sommers, P. 1991, Phys.Rev.Lett., 66, 2697

Tanaka, Y. T., Buson, S., \& Kocevski, D. 2017, The Astronomer's Telegram, 10791

Tavecchio, F., \& Ghisellini, G. 2015, Mon.Not.Roy.Astron.Soc., 451,1502

Torres, D. F., Romero, G. E., \& Mirabel, F. 2005, Chin. J. Astron. Astrophys., 5, S133

Turley, C. F., Fox, D. B., Keivani, A., et al. 2018, arXiv: 1802.08165

Turley, C. F., et al. 2016, Astrophys. J., 833, 117

Ueda, Y., Akiyama, M., Hasinger, G., Miyaji, T., \& Watson, M. G. 2014, Astrophys.J., 786, 104

Urry, C. M., \& Padovani, P. 1995, Publ. Astron. Soc. Pac., 107, 803

Wang, B., \& Li, Z. 2016, Sci. China Phys. Mech. Astron., 59, 619502

Wang, X.-Y., Liu, R.-Y., Dai, Z.-G., \& Cheng, K. S. 2011, Phys. Rev., D84, 081301

Waxman, E., \& Bahcall, J. N. 1997, Phys.Rev.Lett., 78, 2292

-. 2000, Astrophys.J., 541, 707

Wills, B. J., \& Browne, I. W. A. 1986, ApJ , 302, 56

Zhang, B. T., \& Li, Z. 2017, JCAP, 1703, 024 